\title{
Miranda
}

Revue pluridisciplinaire du monde anglophone /

Multidisciplinary peer-reviewed journal on the English-

speaking world

Samuel Beckett : Drama as philosophical endgame?

\section{Circulations de l'écrit : la construction de la communauté catholique anglaise dans les écrits jésuites de 1580 à 1610}

\author{
Gaëlle Serena
}

\section{OpenEdition}

\section{Journals}

Édition électronique

URL : http://journals.openedition.org/miranda/2069

DOI : 10.4000/miranda.2069

ISSN : 2108-6559

\section{Éditeur}

Université Toulouse - Jean Jaurès

\section{Référence électronique}

Gaëlle Serena, «Circulations de l'écrit : la construction de la communauté catholique anglaise dans les écrits jésuites de 1580 à 1610 », Miranda [En ligne], 4 | 2011, mis en ligne le 24 juin 2011, consulté le 16 février 2021. URL : http://journals.openedition.org/miranda/2069; DOI : https://doi.org/10.4000/ miranda.2069

Ce document a été généré automatiquement le 16 février 2021.

\section{cc) (1)}

Miranda is licensed under a Creative Commons Attribution-NonCommercial-NoDerivatives 4.0

International License. 


\title{
Circulations de l'écrit : la construction de la communauté catholique anglaise dans les écrits jésuites de 1580 à 1610
}

\author{
Gaëlle Serena
}

1 L'accession d'Elisabeth Tudor au trône d'Angleterre en 1559 sonne la fin du catholicisme comme religion d'état. Si la reine se défend de stigmatiser son peuple pour sa foi, déclarant qu'elle n'a nullement le désir de sonder l'âme de ses sujets ${ }^{1}$, le lien unissant les catholiques aux puissances étrangères que sont l'Espagne et Rome fait néanmoins problème. L'excommunication de la reine en 1570 ainsi que les rumeurs d'invasion par l'Armada espagnole dès le début des années 1580 placent les catholiques dans une situation délicate. L'arrivée des premiers missionnaires jésuites sur le territoire vient renforcer la méfiance du pouvoir envers ces sujets dont la loyauté est nécessairement partagée entre leur reine et le pape. Les jésuites stigmatisent en effet les peurs d'un gouvernement qui craint de ne pouvoir contrôler l'ensemble de son territoire. Il se crée progressivement deux géographies au sein d'un même royaume: l'une officielle, répressive, craignant le développement d'une force antagoniste en son sein, l'autre clandestine, éclatée et menacée d'effacement.

2 Si cette géographie catholique est encore difficile à cartographier historiquement, cette étude a pour but de montrer que les jésuites ont entrepris sa construction à travers leurs écritures - autobiographies, correspondances mais aussi inscriptions, marginalia et graffiti. Ce sont les modalités de cette construction qui nous intéressent, et plus particulièrement, l'importance de la circulation de ces textes dans la matérialisation d'une communauté que l'on définit à mesure qu'on l'écrit. Mais si le geste d'écrire est indispensable à la survie des catholiques, il en est aussi, à bien des égards, leur arrêt de mort. La circulation de l'écrit est à la fois vitale et mortifère et c'est cette tension contenue dans l'idée même de trace que cette étude se propose d'interroger. 
3 Dès leur arrivée, les jésuites sont la cible des propagandistes protestants, Thomas Bell en particulier :

Note heere Gentle reader, these important obseruations. First, that all Iesuited persons must vow to become traytors. Secondly, that all Iesuites seeke nothing els indeede out their owne lucre and gaine. Thirdlie, that all Iesuited persons must depend vpon the deuill, who as you see heere, inspireth guideth, directeth, and ruleth the Iesuites. From such religion, good Lord deliuer vs. Amen (Bell 68)

Ce sont des chenilles, des parasites qui contaminent le royaume et qu'il convient d'éradiquer ${ }^{2}$. Un certain nombre de textes sont ainsi promulgués à cet effet ${ }^{3}$. Mais la méfiance suscitée par les missionnaires se propage vite à l'ensemble de la communauté. En 1593 est ainsi promulguée une nouvelle loi, The Act against the Recusants, imposant de nouvelles restrictions aux réfractaires, ces catholiques qui refusent de se rendre au service protestant, qui n'ont dès lors plus le droit de circuler librement à l'intérieur du royaume ${ }^{4}$.

5 Mais il ne s'agit pas seulement de confiner les catholiques dans un espace contrôlé, il faut aussi effacer les traces d'une identité commune. Les premières injonctions de 1559 interdisaient déjà les signes catholiques:

[The clergy is to] take away, utterly extinct and destroy all shrines, covering of shrines, all tables and candlesticks, trundles or rolls of ware, pictures, paintings and all other monuments of feigned miracles, pilgrimages, idolatry and superstition, so that there remain no memory of the same in walls, glasses, windows or elsewhere within their churches or houses. And they shall exhort all their parishioners to do the like within their several houses ${ }^{5}$.

6 A partir des années 1580, la situation des catholiques s'aggrave. Toute la paraphernalia papiste, jusque-là tournée en dérision ${ }^{6}$, devient offense sérieuse, la marque tant recherchée d'une adhésion coupable au culte papiste, comme l'indique la remarque d'Edward Hastings à Lord Burghley, « A reconciled papist may be known by refusing to go to church and wearing a crucifixe, Agnus Dei, or grana benedicta which are not given until reconciliation ${ }^{7}$. " Les archives révèlent par ailleurs que les fouilles des maisons catholiques ont été plus nombreuses à partir des années 1580 et les inventaires, listant les objets catholiques trouvés sur place, bien plus fréquents ${ }^{8}$. Il semble donc bien que ce soit la religion catholique dans son ensemble, et non les seuls missionnaires, à laquelle s'attaque à présent le pouvoir. Dans de nombreux comptes-rendus d'interrogatoires, la seule présence de ces objets suffit à incriminer son détenteur. Le 26 janvier 1601-2, Richard Barret est arrêté et interrogé pour la seule possession d'un crucifix en cuivre 9 . S'il est par la suite relâché, son arrestation confirme le rapport étroit qu'établit l'autorité entre catholicisme et sédition.

7 Etre catholique n'est plus une conviction religieuse mais une soumission délibérée à des puissances étrangères et donc un acte de trahison aux yeux des autorités qui cherchent à imposer ce discours en marquant les catholiques jusque dans leur chair. Un jeune étudiant est en effet marqué au fer rouge, sort réservé aux criminels, pour avoir refusé d'abjurer sa foi: « When even so there was no conquering him, they branded him ${ }^{10}$ - that is to say, pierced his ear with a hot iron, and so let him go " (Hicks 77). Les récusants sont dès lors relégués au rang de hors-la-loi. Edmond Campion doit lui aussi porter la marque de l'infamie sur le trajet qui l'amène à Londres après son arrestation. Les gardes fixent en effet sur son chapeau un morceau de papier sur lequel est écrit «Campion, the seditious Jesuit». Lors des exécutions, il devient primordial pour les 
autorités de contrôler l'image du condamné. C'est pourquoi le bourreau placarde des affiches sur l'échafaud rappelant les crimes du supplicié :

No Priests are suffred to speak at their deathes, but so soone as they are dead, Topcliff, in an oration unto the people, faineth the cause to be for assisting the intended invasion of the realme; and to that extent he fixeth also papers upon the gallows or gibbett. (Verstergan 40)

La communauté catholique est donc menacée d'éclatement, voire d'effacement. Sans lieux où vivre ensemble, cette communauté est condamnée à la dissolution. Il devient alors vital pour les catholiques de laisser une trace sur cette table rase afin que d'autres puissent la lire et se reconnaître en elle.

Ecrire, c'est tout d'abord poser la présence du sujet. Dans un contexte où l'identité catholique est sans cesse niée, l'enjeu n'est pas des moindres. Pour ces hommes que l'on cherche à réduire au silence, l'écriture devient geste ontologique. Elle permet de réaffirmer une identité qui se dérobe. Les autobiographies, écrites par les jésuites John Gerard et William Weston à la demande de leurs supérieurs ${ }^{11}$, sont autant de mises en scène de leurs auteurs qui construisent ainsi leur personnage en essayant de lui donner une cohérence. L'entreprise autobiographique permet en effet la reconstruction d'un sujet mis à mal par une vie de dissimulation sous des déguisements, des faux noms ou au fond de ces caches creusées au cœur des demeures catholiques ${ }^{12}$.

Les graffiti, encore visibles sur les murs de la Tour de Londres, attestent eux aussi le besoin impérieux des prisonniers à se dire. Les noms et monogrammes qu'ils ont gravés dans la pierre rappellent à nous, lecteurs, la présence spectrale de ces hommes soustraits au regard des autres. Ces graffiti peuvent n'être qu'un simple nom ou un dessin. Certains semblent avoir été gravés à la hâte, d'autres sont plus élaborés mais tous attestent l'importance de laisser une marque sensible dans un lieu censé déposséder le prisonnier de son identité mais qui en devient paradoxalement le dépositaire ${ }^{13}$. 


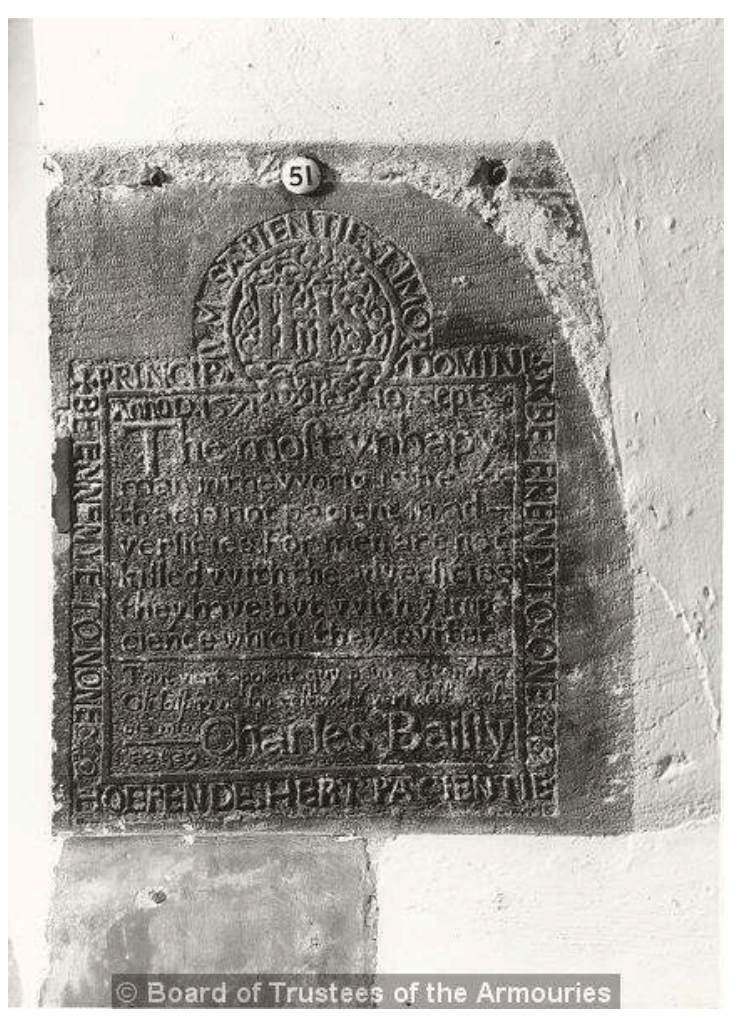

11 La trace, qu'elle soit nom, symbole ou récit, contient et fait vivre celui qui la produit. Elle demeure présence, assertion de soi et permet ainsi de lutter contre l'effacement du corps.

Ecrire, c'est donc une expérience de soi, on écrit pour se dire mais c'est aussi une expérience de l'autre, expérience vitale dans un contexte où les liens sont usés par l'exil, l'emprisonnement et la mort. Laisser une trace, quelle qu'elle soit, c'est établir une communication dans l'absence, c'est espérer être lu et donc poser la présence de l'autre. C'est en ce sens que l'écriture est vitale aux prisonniers car, sans la possibilité d'un échange, la prison devient sépulcre. Certains cachots étaient d'ailleurs appelés « les limbes ». ${ }^{14}$ Pour échapper à cet isolement mortifère, les prisonniers ont recours à l'écrit qui n'est plus seulement assertion de soi mais aussi reconnaissance de l'autre comme lecteur potentiel. Le geste d'écrire permet ainsi au prisonnier de s'inscrire dans un circuit, réel ou fantasmé, mais dynamique malgré le confinement et la séparation. Le support importe peu tant que l'écriture reste possible. John Gerard raconte ainsi dans son autobiographie comment l'un de ses amis, privé d'encre et de feuilles, se met à griffonner sur le moindre morceau de papier qu'il trouve :

Since he had been closely confined and had been deprived of ink, he had scribbled most of the book in pencil on loose scraps of paper, and as he completed each part he sent it to me to look over and correct any mistakes in doctrine. (Gerard 89)

Ces gribouillages permettent au prisonnier de tisser des liens avec le monde extérieur dont il n'est pas encore totalement exclu. Même le graffiti, a priori figé et intime, raconte une histoire destinée à un lecteur futur. Certaines inscriptions ont valeur de maximes adressées aux prochains prisonniers. L'inscription laissée par Philip Howard semble vouloir réconforter ceux qui partageront le sort de son auteur: «The more 
affliction (we endure) for Christ in this world / The more glory (we shall obtain) with Christ in the world to come. » (Barter 29)

Figure 2. Inscription de Philip Howard, Tour Beauchamp, 1587

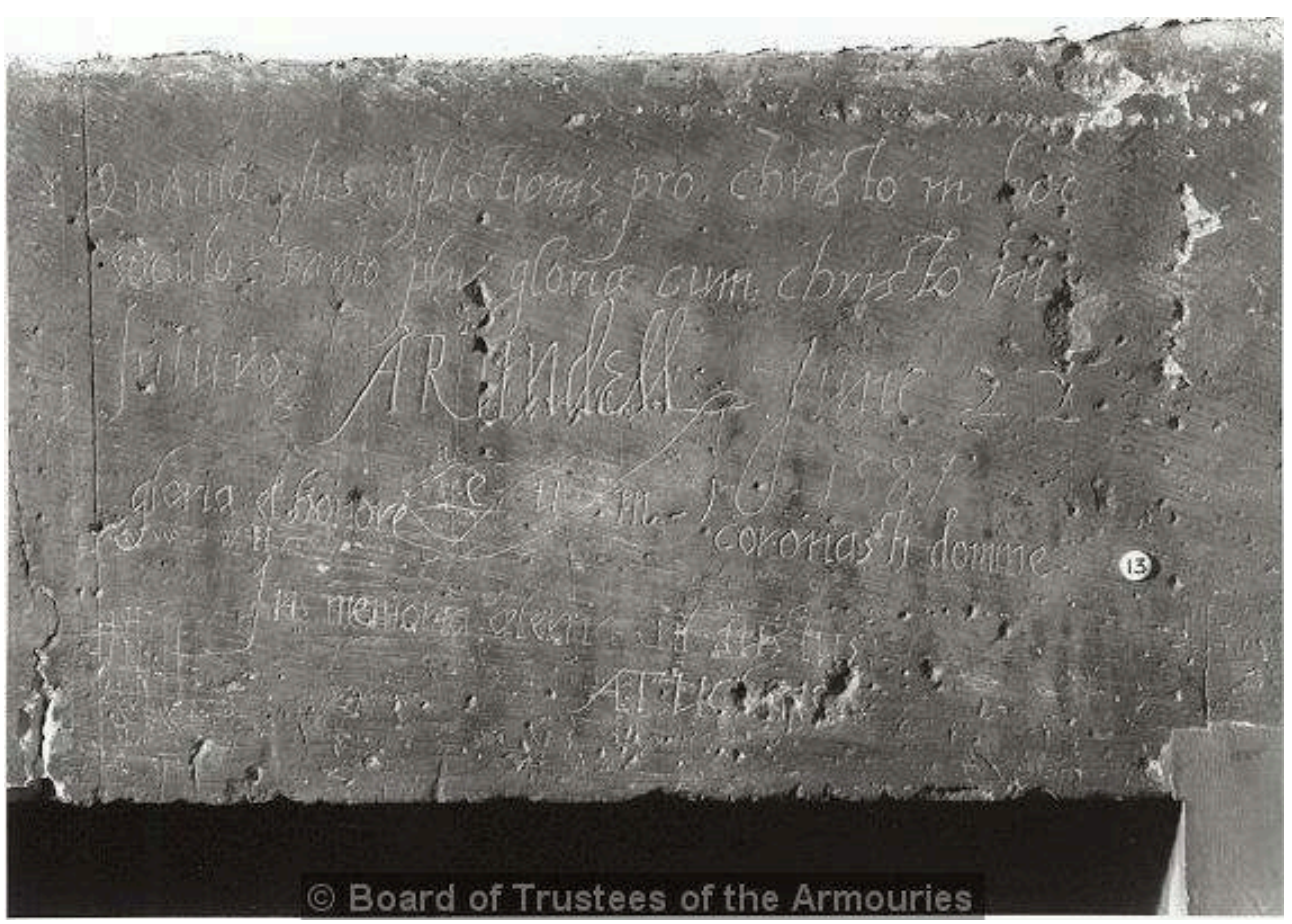

Cette même inscription est d'ailleurs commentée par un autre prisonnier, incarcéré après la mort de Philip Howard: "Thou has crowned him with honour and glory, 0 Lord / The just shall be held in eternal remembrance." (Barter 29). Cet échange posthume illustre l'idée selon laquelle le graffiti n'est plus seulement un geste intime qui dit la présence du sujet mais qu'il s'agit aussi d'un geste résolument orienté vers l'autre. La trace est en effet lue, interprétée et racontée. Le graffiti circule alors pour réapparaitre dans d'autres textes. L'inscription gravée par le jésuite Henry Walpole sur les murs de la Salt Tower ${ }^{15}$ réapparait dans le récit de Gerard :

15 The next morning I walked round my cell. In its dim light I found the name of the blessed father Henry Walpole cut with a chisel on the wall (...) It was a great comfort to me to find myself in a place sanctified by this great and holy martyr, and in the room where he had been tortured so many times. (Gerard 104)

L'inscription bénéficie donc d'une circulation plus large que prévue. Elle résonne audelà des simples murs de la cellule. Bien plus qu'un simple sillon, elle devient signe à déchiffrer, élément d'un langage qui met en présence auteur et lecteur. L'écriture représente ainsi un enjeu vital dont l'acte permet de se dire - j'écris donc je suis mais aussi d'affirmer l'existence de l'autre - j'écris donc tu es.

Cette double tension propre à l'écrit trouve sa meilleure expression dans l'échange épistolaire qui réunit émetteur et destinataire au cœur même de l'écriture. Les jésuites font leur le culte humaniste d'une amitié fondée sur l'échange de lettres. Dans l'ouvrage Regulae Societatis Iesu publié en 1561, pas moins de trente-cinq articles sont consacrés au bon usage des lettres ${ }^{16}$ L'entretien d'une correspondance régulière était une obligation pour les membres de l'ordre dispersés à travers le monde. Les Provinciaux devaient écrire une fois par mois à leurs supérieurs qui devaient écrire 
tous les trois mois aux Généraux. L'étude de ces lettres montre que les jésuites attachent une réelle importance à ces échanges considérés comme indispensables au maintien d'une réelle amitié. Dans une lettre qu'il écrit à un ami perdu de vue, Robert Southwell souligne le rôle fondamental de l'échange épistolaire dans le tissage et la préservation des liens malgré l'absence et la séparation :

I decided (...) to have done with drowsy silence, and to place on a candlestick the light of our friendship so long hidden under a bushel (...) I was proceeding to carry out in deed what I had conceived in thought, when suddenly a letter from you was handed to me, on perusing which I discovered that you had written to me in the very same frame of mind. Regarding this as an interposition of God's providence, who would not allow the union between us, (...), to be dissolved, but rather willed to see it fostered, revived, strengthened by mutual interchange of letters ${ }^{17}$.

Quand ils ne peuvent pas écrire, les jésuites prennent grand soin à s'en excuser, expliquant précisément pourquoi ils n'ont pu donner de nouvelles plus tôt. Les lettres s'ouvrent souvent sur le rappel d'une lettre passée, ce qui permet d'ancrer la correspondance dans un échange régulier et dynamique. On écrit même parfois pour ne rien dire, seulement pour renforcer le lien avec l'autre. Ce n'est pas tant le contenu que la lettre elle-même qui importe ici. L'écriture devient objet dont la seule circulation permet de nouer des liens entre émetteur et destinataire.

L'écriture ainsi réifiée circule de mains en mains et dessine les contours de la communauté. La circulation des lettres ou des textes manuscrits tisse des liens entre plusieurs personnes : l'auteur, le scribe parfois, le messager et les destinataires. Ce sont des objets malléables qui gardent la trace de ceux qui les manipulent. Ils prennent alors la forme de véritables palimpsestes, de textes à plusieurs mains, figeant ainsi sur la page les liens qui unissent les différents auteurs. Nombreux sont les textes dont les marges portent la marque d'un intermédiaire qui vient annoter le texte original.

Les conditions même de la circulation de ces lettres matérialisent les liens entre les différents membres de la communauté. Une même lettre peut être destinée à des personnes différentes ou redistribuée après sa lecture. Plusieurs lettres sont parfois contenues dans un seul et même paquet bien que pour des destinataires différents. L'échange matériel de ces lettres contribue donc à former ce que Harold Love appelle "a scribal community" (Love 117). La circulation de ces lettres par la copie notamment, ou la lecture à voix haute, permet de tisser des liens solides entre les différents points du cercle ainsi formé. C'est par un échange de lettres entre Edmond Campion et le général Aquaviva que John Gerard connaît son voisin de cellule, Ralph Emerson, qu'il n'a, par ailleurs, jamais rencontré : «Next door to me was Ralph Emerson, the Brother, who was referred to by Father Campion in a letter to Father General as 'My little man and I' » (Gerard 78).

21 La circulation de ces textes n'est pas toujours réservée à l'intimité du cercle communautaire. Certains textes étaient destinés à une circulation plus large, comme un défi direct aux autorités. Un catholique qui se faisait appeler Greenlowe, fut arrêté en possession d'une lettre destinée à la reine, lettre qu'il comptait copier en de nombreux exemplaires et placarder dans des endroits publics. John Gerard, accusé d'avoir pris part au Complot des Poudres, rédige lui aussi une lettre qu'il destine, a priori, à un ami mais dont il fait plusieurs copies qu'il disperse dans les rues de Londres. Mais si la lecture publique de ces écrits était ici recherchée, ce n'était évidemment pas toujours le cas et les textes à l'étude témoignent de l'inquiétude qui entoure la production de ces signes dont les lectures sont nécessairement multiples. 

la lettre qu'il écrit à Robert Parsons en mars 1592: « Some other things I could signifie, which for want of more secure means of wryting I will omite » (Pollen 208). Un rapide détour par les arcanes du pouvoir élisabéthain nous permet de comprendre l'inquiétude de ces hommes. Les maîtres-espions de la reine, Lord Burghley et Sir Francis Walsingham, ont en effet mis en place un système de surveillance particulièrement redoutable car bien que peu nombreux, les jésuites semblent hanter l'imaginaire des autorités dont la priorité devient le contrôle de ces agents nomades et invisibles envoyés par l'ennemi. William Weston se plaint de cette surveillance renforcée: «Every road, cross-way and port was watched day and night, and sealed off so effectively that no person could pass without the most rigorous examination " (Weston 93). Southwell écrit "Such is the multitude of spies, that we cannot set foot out of doors, nor walk in the streets, without danger to our lives $»^{18}$.

Ces espions infiltrent également la communauté catholique et parasitent le réseau de communication mis en place par les jésuites. Anthony Rivers évoque, dans l'une des ses lettres, le catholique Barwis qui est mystérieusement libéré sur ordre de la reine et sera en fait envoyé comme agent double à l'étranger ${ }^{19}$. Il mentionne aussi le prêtre Barneby qui a été placé en prison afin d'intercepter les lettres envoyées aux prisonniers ${ }^{20}$. Dans de telles circonstances, la trace, pourtant vitale, devient extrêmement dangereuse.

Une fois tombés entre des mains ennemies, les signes sont en effet soumis à une toute autre lecture. Jusqu'alors signes identitaires ou marques de solidarité, ces écrits deviennent les signes infamants de la trahison. Ce qui était communauté devient un ensemble de relations coupables. Les lettres, en particulier, peuvent être extrêmement incriminantes. La seule possession d'une lettre écrite par un catholique peut avoir des conséquences graves et nombreux sont ceux qui refusent cette association sulfureuse, comme l'atteste la lettre qu'écrit Thomas Copley à Lord Burghley: «I send this by the ordinary post, because I see such a terror generally stricken into the hearts both of my friends and servants there, as not one of them writes to me, (...) nor dares deliver any letter I send over. $»^{21}$

Porter une lettre écrite par un catholique était déjà en soi un délit. Si cette lettre comportait quelque information confidentielle, l'interception pouvait alors s'avérer fatale. Dans une lettre à Robert Southwell, le Général Aquaviva ${ }^{22}$ souligne le danger que pouvait représenter la confiscation de certaines informations: « That the third [letter] which you wrote has gone astray, causes us real sorrow, for we apprehend it was such a character that, supposing it fell into hostile hands, evil results might be feared.» (Pollen 320). Les compte-rendus de procès nous éclairent sur ces conséquences funestes. Les preuves produites au procès de Philip Howard, par exemple, sont les lettres qu'il a écrites au Cardinal Allen et qui démontrent, pour les autorités, les liens coupables que le comte entretient avec les puissances étrangères.

Mais le contenu n'est pas le seul enjeu. Le caractère profondément métonymique de la trace, s'il permet de donner vie à son auteur, le met également en danger. La trace matérialise et fige les liens unissant l'objet et celui qui y a apposé sa marque. Elle désigne donc son auteur et l'expose aux yeux des autorités. La culpabilité de Philip Howard est prouvée, non seulement par sa correspondance avec William Allen, mais aussi par un petit bout de papier retrouvé dans ses affaires et qui a servi à protéger les perles d'un chapelet. Sur ce papier est écrit le nom du comte et la seule proximité entre l'objet interdit et le nom suffit à dire la culpabilité de l'accusé. ${ }^{23}$ De la même façon, John 
Gerard manque de se faire arrêter lors d'une fouille, car les notes qu'il a laissées derrière lui trahissent sa présence : «They knew now (...) I had been hiding in the same house. They had my notes as proof of it, for there was no doubt they were mine.» (Gerard 155). Un de ses serviteurs se dénonce à sa place, car il n'a pas d'autre choix s'il veut sauver le prêtre: «There's no one to own the books and papers in the room, for they're after you. If there's no owner, they won't stop till they've found you. » (Gerard 153).

27 L'écriture, comme prolongement du corps, devient donc signe accablant d'une présence illicite. Les mots s'empressent de trahir la main qui les a formés. John Gerard explique comment il a floué Richard Topcliffe, le tortionnaire de la reine, en modifiant son écriture :

He was hoping to trip me up in what I wrote, or at least, to get a sample of my handwriting. If he had this he could prove that certain papers found in the search of the houses belonged to me. I saw the trap and wrote in a feigned hand. (Gerard 69)

Les deux hommes se livrent alors un combat féroce pour contrôler ce qui va finalement apparaître sur la page. John Gerard explique qu'il ne laisse pas d'espace entre le corps de la lettre et sa signature afin que Topcliffe ne puisse rien rajouter. L'espace de la page doit être absolument maîtrisé sous peine d'être envahi par l'autre :

'I'll write the truth or nothing,' I said. 'No,' he snarled, 'write such and such, and I will make a fresh copy of what you write.' 'I shall write what I want and not what you want. If you like, you can show what I've written to the Council. All I am going to add is my signature.' Then I signed very close up to the line so that he had no space to add anything. He saw he was beaten and in his frustration he blurted out threats and blasphemies. (Gerard 70)

Ces exemples nous montrent à quel point il devient vital pour les catholiques de contrôler cette trace qui, à peine créée, ne leur appartient déjà plus. Conscients de cet enjeu, les jésuites développent une véritable culture du secret qui vient nourrir l'image pour le moins interlope de l'ordre. Si cette image est évidemment exagérée dans les discours de propagande, il n'en reste pas moins que les jésuites, fervents partisans de l'équivocation, maîtrisent à merveille l'art de la dissimulation.

Une des premières règles est de contrôler la circulation du texte, de le cacher aux yeux de l'ennemi. Pour ce faire, les jésuites ont recours à des stratagèmes toujours plus ingénieux, dissimulant des lettres compromettantes dans des boutons ou des cachettes spécialement prévues à cet effet. John Gerard explique comment il fait circuler des messages depuis sa cellule: «Then I wrapped the paper round one or two collars to make it look as if it was being used to keep the collars clean.» (Gerard 92) Il écrit aussi sur des papiers dont il se sert pour envelopper les chapelets qu'il a confectionnés à partir de pelures d'orange et qu'il offre à d'autres prisonniers. On note d'ailleurs chez Gerard un certain plaisir à raconter comment il parvient à déjouer la vigilance de son gardien. On s'aperçoit en lisant son autobiographie que ce n'est pas tant le message en lui-même qui importe que la possibilité de cette circulation, de cet échange entre l'espace soi-disant clos de la cellule et le monde extérieur.

31 Une autre technique consistait à dissimuler un message écrit à l'encre invisible entre les lignes d'un texte qui n'était alors qu'un leurre. John Gerard explique que le jus de citron permet de créer une trace qui ne se révèle qu'aux yeux des initiés: «I had written in lemon juice, so that no writing should appear on the paper » (Gerard 93). Il suffisait ensuite de passer le papier près d'une source de chaleur pour faire apparaître 
le message. Il précise néanmoins que le jus d'orange est préférable dans la mesure où, une fois révélé, le texte écrit ne s'efface plus et permet donc au véritable destinataire de savoir s'il a déjà été lu :

A letter in orange juice cannot be delivered without the recipient knowing whether or not it has been read [...] and in this way I got all the information I needed from them, and they received the spiritual help they sought from me. (Gerard 119)

Toutes ces techniques, et bien d'autres, comme le recours à de faux frontispices pour éviter la censure ou de fausses adresses, permettaient d'assurer la bonne circulation du texte et d'éviter son interception.

Mais si le texte était effectivement intercepté, il fallait faire en sorte qu'il reste illisible. Dans sa lettre au jeune et impétueux Robert Southwell, le Général Aquaviva rappelle à son élève les rudiments de l'écriture secrète. Il lui demande d'être plus prudent: « your Mastership should be warned that if in the dispatch of letters you cannot exercise greater caution (as we readily believe to be the case), you should at least in writing them be more on your guard» (Pollen 320). Il y préconise l'utilisation d'un langage allégorique permettant de dissimuler le véritable sens du texte :

Do not say so much in plain and open terms, lest (if what you write fall into others' hands) danger should thence arise either to others or to yourself; [...] Things, especially when of importance, should be somewhat veiled in allegory (for the receiver will grasp the sense) and when persons are in question, they should be merely alluded to indirectly. (Pollen 320)

Le reste de la lettre est une mise en pratique de ces conseils. Seuls les prénoms sont donnés, «persecutors » devient " creditors ", Pound et Metham deviennent «Weight » et «Winning-post », les calices ne sont plus que des «cups » et la mission est évoquée sous le terme de « trade».

Les jésuites développent donc une série de tropes leur permettant d'évoquer sans crainte les affaires en cours. Ils ont souvent recours aux métaphores agricoles pour parler de leur mission. Dans une de ses lettres à Richard Verstergan, Henry Garnet écrit "We are lyke to have heare a plentifull yeare, so that we may make great comoditie of corne, yf we be secret in our course», "corn» signifiant ici "conversions». (Verstergan 67) Ils utilisent aussi des codes comme dans cette lettre de Verstergan à Persons datée du 6 août 1592 :

Whereby it may please God that some good opportunity may fall oute for $208 \mathrm{yf}$ the occasion be taken on; and in my slender judgment, it were good that the $7 \mathrm{~m} 14 \mathrm{p}$ me 20 that cometh from 38 having delivered their charge in 20 might foorthwith come into 54 which may fall oute to especiall good purpose, yf some thinges do succede in 25 that I shall not nede to name. I beseech you to consider hereof. (Verstergan 63)

Ces codes permettent de protéger le secret de l'information et l'identité des correspondants. La lettre ainsi chiffrée reste lettre morte aux yeux du lecteur intrusif. L'écriture est bien visible mais elle reste illisible. Le partage de ces codes permet de renforcer la communauté autour d'un savoir connu idéalement d'eux seuls. L'espion Thomas Rogers écrit ainsi à Walsingham: «The enclosed is the last from Rome. I do not know the contents of the cipher, but I have set it down verbatim. " L'espion se situe donc résolument à l'extérieur du cercle communautaire dont la limite est le langage codé. Le secret joue un rôle important dans la cohésion du groupe ici. Il permet aussi, dans une certaine mesure, de déstabiliser l'ennemi. Samuel Harsnett écrit, en parlant des pseudonymes qu'utilisaient les jésuites : 
Or else it may seeme that our vagrant deuils heere did take theyr fashion of new names from our wandring Iesuits, who to dissemble themselues, haue alwaies three, or foure odde conceited names in their budget: [...] Or else there is a confederation between our wandring Exorcists, and these walking deuils, and they are agreed of certaine vncouth non-significant names, which goe currant amongst themselues, as the Gipsies are of gibridge, which none but themselues can spell without a paire of spectacles. (Harsnet 46)

Le recours à ces stratégies de dissimulation est donc nécessaire, bien souvent salutaire mais aussi périlleux. Ces stratégies peuvent en effet être découvertes et détournées, les catholiques devenant alors les artisans de leur propre ruine. Car les autorités ne font pas qu'intercepter les lettres, elles intègrent le circuit et le détournent afin de piéger émetteurs et destinataires. Dans son récit du Complot des Poudres, le jésuite Oswald Tesimond relate comment son ami Henri Garnet, alors accusé d'avoir fomenté le complot, s'est fait piéger à son propre jeu :

Father Garnet now wrote a letter to a priest. [...] Garnet asked him for certain necessary items which he needed. In the margin of the letter, which was written in ordinary ink, he wrote with orange or lemon juice what had passed at his frequent examinations. [...] [The gaoler] took it to the lieutenant of the Tower. He warmed it at the fire and read all that was written in the margin. But the letter was now scorched and discoloured by the fire so that it could not be sent on. They therefore wrote it out again, counterfeiting the original hand, so that when Garnet received his reply he would be more convinced of the pretended fidelity of his gaoler. (Tesimond 185)

Les lettres codées attirent nécessairement l'attention et sont bien souvent déchiffrées ${ }^{24}$, elles deviennent alors preuves à conviction. L'affaire Babington est sans doute la plus célèbre. En 1585, Marie Stuart est placée sous la garde de Sir Amias Paulet. Tout échange épistolaire est apparemment interrompu mais Walsingham encourage la circulation clandestine de lettres entre Marie, Babington et Thomas Morgan. Ses lettres sont bien sûr interceptées et déchiffrées avant d'être portées aux destinataires. Persuadés que le moyen d'acheminement des lettres est sûr, les catholiques sont en fait en train d'écrire les preuves qui les mèneront à l'échafaud. Marie scelle son destin, le 17 juillet 1586, lorsqu'elle donne son accord au plan de Babington visant à la libérer.

$\mathrm{Au}$ cœur des textes jésuites repose ainsi le paradoxe indépassable d'une écriture qui est à la fois vitale, essentielle à la construction d'une communauté que l'on cherche à faire disparaître mais aussi profondément mortifère dans la mesure où la circulation de cette trace n'est jamais totalement contrôlée, la lecture des signes nécessairement multiple.

Une fois découverts, les condamnés évoluent sur une scène contrôlée par les protestants. Pourtant, ils n'en cessent pas moins d'écrire malgré les efforts des autorités pour les réduire au silence. L'échafaud devient espace agonistique que les catholiques, privés de parole, cherchent à investir. L'écrit se fait alors relais de la voix. Certains condamnés sachant qu'ils seront interrompus par le bourreau écrivent en effet leur défense sur des feuilles qu'ils jettent dans la foule :

As it was impossible now to give a complete and detailed explanation of his conduct, he took out a sheaf of papers from his jacket. These contained a full statement of his case, and he scattered them among the crowd so that they would have the chance to snatch and read for themselves all they had been prevented from hearing. (Weston 103) 
Et c'est peut-être sur l'échafaud que naît la possibilité d'une trace enfin univoque car figée dans le sang des martyres. C'est ce que semble dire John Boste qui, avant de mourir, lance aux autorités :

Although I am now to be deprived of liberty, my blood withal and death and innocence, shall preach in the hearts of those whom God will call and gather to his Holy Catholic Church. My head and quarters will preach every day on your gates and walls the truth of the Catholic faith. (Pollen 286)

\section{BIBLIOGRAPHIE}

Calendar of the Manuscripts of the Most Hon. the Marquis of Salisbury. Preserved at Hatfield House, Hertfordshire. R. A. Roberts (ed.). vol. 12. 1602. London: His Majesty's Stationery Office, 1910. Barter, Sarah. Treasures of the Tower: Inscriptions. London: Her Majesty's Stationery Office, 1976. Bell, Thomas. The Anatomie of Popish tyrannie ... of Secular Priests and English hispanized Iesuites, with their Iesuited Arch-Priest. London: [Iohn Harison] Richard Bankworth, 1603.

Boswell, Grant. « Letter Writing among the Jesuits: Antonio Possevino's Advice in the 'Bibliotheca Selecta' " Studies in the Cultural History of Letter Writing, Huntington Library Quarterly, 66:3/4 (2003): 247-262.

Foley, Henry. Records of the English Province of the Society of Jesus, London: Burns and Oates, 1875. Gerard, John. The Autobiography of an Elizabethan, 1609. Philip Caraman trans. London: Longmans, Green and Co., 1951.

Harsnett, Samuel. A Declaration of egregious popish impostures, London: James Roberts, 1603.

Hicks, Leo. Letters and Memorials of Fr. Persons. London: Catholic Record Society, 1942.

Love, Harold. Scribal Publication in Seventeenth-Century England. Oxford: Clarendon Press, 1977.

Pollen, John Hungerford. Unpublished Documents relating to the English Martyrs. Series v. 5, 2.

London: Catholic Record Society, 1908.

Tesimond, Oswald. The Gunpowder Plot, The Narrative of Oswald Tesimond alias Greenway, Francis Edwards trans. London: The Folio Society, 1973.

Verstergan, Richard. The Letters and Despatches of Richard Verstergan. Anthony G. Petti (ed.). Series LII. London: Catholic Record Society, 1959.

Weston, William. The Autobiography of an Elizabethan, 1611. Philip Caraman trans. London: Longmans, Green and Co., 1955.

\section{NOTES}

1. La phrase restée célèbre est issue d'un pamphlet de Francis Bacon, Certain Observations upon a libel (1592), dans lequel il écrit « her majesty not liking to make 
windows into men's hearts and secret thoughts (...) tampered her law ", The Works of Francis Bacon. London: 1824, Vol. 3. 73.

2. William Vaughan écrit une lettre au Conseil dans laquelle il compare les jésuites à des chenilles : «I thought it the part of her Majesty's loyal subject in these my travels to forewarn the Council of certain caterpillars, I mean Jesuits and seminary priests, who [...] are to be sent from the English seminary at Valladolid", Calendar of the Manuscripts of the Most Hon. the Marquis of Salisbury, Preserved at Hatfield House, Hertfordshire. Ed. R. A. Roberts. Vol. 12: 1602, London: His Majesty's Stationery Office, 1910, 211.

3. En 1585, est promulgué The Act against Jesuits and Seminarists : «It shall not be lawful to or for any Jesuit, seminary priest, (...) to come into, be, or remain in any part of this realm, (...) ; and if he do, that then every such offence shall be taken and adjudged to be high treason; and every person so offending shall for his offence be adjudged a traitor, and shall suffer, lose, and forfeit, as in case of high treason ». (27 Elizabeth, Cap. 2, March 1585). La proclamation royale de 1591 vient réaffirmer la méfiance du pouvoir à l'égard de ces agents envoyés par Rome.

4. The Act Against Recusants, 35 Elizabeth, Cap. 2, 1593: «For the better discovering and avoiding of all such traitorous and most dangerous conspiracies (...) by sundry wicked and seditious persons, who, terming themselves Catholics, and being indeed spies and intelligencers, (...) do secretly wander and shift from place to place within this realm, to corrupt and seduce her majesty's subjects, and to stir them to sedition and rebellion: $\mathrm{Be}$ it ordained and enacted (...) that every person above the age of sixteen years, born within any of the queen's majesty's realms and dominions, or made denizen, being a popish recusant, (...) shall within forty days (...) repair to their place of dwelling where they usually heretofore made their common abode, and shall not, any time after, pass or remove above five miles from thence. »

5. Henry Gee and W.H. Hardy (eds.). Documents Illustrative of English Church History, New York: 1896, 428.

6. Le Vice-Chancelier Byng qualifie ces objets de "popish trinkets", CSP, Elizabeth, Lansdowne. Dec 14 (1572). 64. Vice-Chancellor Byng to Lord Burghley, 30.

7. CSP, Elizabeth, Domestic, 1591-1594. April 20 (1591). 126. Edward Hastings to Lord Treas. Burghley, 27.

8. Pour des exemples d'inventaires, voir CSP, Elizabeth, Domestic, 1581-1590. Dec 10 (1583). 14. The Bishop and Mayor of Winchester, and others of the city, to Walsyngham, $135 \mathrm{ou}$ CSP, Elizabeth, Domestic, 1581-1590, Jan 26 (1584).47. An inventory of the Books and other Popish relies found in the house of Mistress Hampden of Stoke in the county of Buckingham, 155.

9. CSP, Elizabeth, Hatfield, Jan 26 (1601-2). 58. William Bramble, Mayor of Poole, and William Hiley, Preacher of the Word, to Sir Robert Cecil, 32.

10. Je souligne.

11. Gerard, John, The Autobiography of an Elizabethan, 1609 et Weston, William, The Autobiography of an Elizabethan, 1611.

12. Les catholiques aménageaient en effet des caches au cœur même de leurs maisons, derrière des panneaux de bois ou dans des cheminées, pour permettre aux prêtres qu'ils accueillaient, de se cacher pendant les fouilles des poursuivants. Nicholas Owen, principal architecte de ces caches, fut d'ailleurs l'un des seuls laïcs, avec George Gilbert, 
à être admis dans la Société de Jésus à cette époque, en raison des services rendus à l'ordre. Voir Peter Davidson, «Recusant Catholic Spaces in Early Modern England », in Catholic Culture in Early Modern England. Ronald Corthell, Frances E. Dolan, Christopher Highley and Arthur Marotti (eds.). Notre Dame: University of Notre Dame Press, 2007.

13. Voir à ce sujet les articles de Véronique Plesch, «Memory on the wall: graffiti on religious wall paintings ", Journal of Medieval and Early Modern Studies. Vol. 32: n ${ }^{\circ} 1$ (Winter 2002) 167-197, et Jason Scott Warren, «Reading Graffiti in the Early Modern Book ", The Huntington Library Quarterly. vol.73: $n^{\circ} 3$. (2010) 363-381. Pour une analyse des graffiti dans la Tour de Londres sous le règne d'Henri VIII, voir l'article de Ruth Ahnert, "Writing in the Tower of London during the Reformation, ca. 1530-1558 ", Huntington Library Quarterly. Vol.72 : n² (2009) 168-192.

14. Southwell écrit dans une de ses lettres au général de la Société Claudio Aquaviva, datée du 31 août 1588 : «Felton too was flogged at Bridewell ; and afterwards he was thrust into that most darksome Dungeon at Newgate called Limbo, and kept there in chains and shackles for 15 weeks. » (Pollen, 326).

15. http://collections.royalarmouries.org/viewItem.php?i=346632 visité le 29 mai 2011.

16. Boswell, 247-262.

17. Lettre de Robert Southwell à John Deckers, octobre 1580 (Pollen, 294).

18. Lettre de Southwell à Claudio Aquaviva datée du 21 décembre 1586 (Pollen, 314).

19. Anthony Rivers écrit à Ridolfo Perino, alias Parsons, « Barwis hath liberty for three months by the express command of Her Majesty, it is thought he hath promised some special service », Foley, 1875, 42.

20. "Barneby lieth at the Clink, and receiveth ordinary packets of letters from Bagshawe by every post, which he carrieth to the Bishop of London. He is in prison, but placed there of purpose to that end », Foley, 1875, 32.

21. Lettre de Thomas Copley à Lord Burghley, CSP, Elizabeth, Domestic, Addenda 1566-1579. Vol. XXIII, 9. Feb. 261573.

22. Claudio Aquaviva fut le supérieur général des jésuites de 1581 à 1615 . Voir « Claudio Aquaviva. » Encyclopædia Britannica. Encyclopædia Britannica Online. Encyclopædia Britannica, 2011. Web. 18 May. 2011.

<http://www.britannica.com/EBchecked/topic/31130/Claudio-Aquavivas.

23. De nombreuses archives traitant du procès de Philip Howard ont été publiées dans Pollen, John, The Ven. Philip Howard, Earl of Arundel, 1557-1595 : English martyrs, vol. II, 1919.

24. Les archives offrent de nombreux exemples de lettres interceptées et déchiffrées par Thomas Phelippes. Voir par exemple la lettre écrite par Richard Verstegan à Roger Baynes, CSP, Elizabeth, Domestic, 1595-1597, Vol.CCLII, 15. May 10/20 1595. Les annotations de Phelippes apparaissent très clairement dans la marge du manuscrit. 


\section{RÉSUMÉS}

Cette étude se propose d'interroger la place de l'écrit et des actes d'écriture dans la construction d'une communauté réduite au silence, la communauté catholique sous le règne d'Elisabeth Tudor. A la fois moyen d'information et geste ontologique, l'écrit représente un enjeu vital pour résister à l'effacement. La circulation des textes permet de tracer les contours d'une communauté dont l'existence est étroitement liée à la production de l'écrit. Mais, si elle détournée, la trace peut alors devenir arrêt de mort, révélant l'identité de celui qui l'a produite aux yeux de l'intrus qui la déchiffre.

The purpose of this study is to analyze to what extent the act of writing helped define the catholic community under Elizabeth I. As both a means of information and an ontological process, writing was vital to the Catholics who were forced underground. By circulating texts throughout the country and in the rest of Europe, the Jesuits shaped a community highly reliant on written material. But writing was also incredibly dangerous as it marked the author as irredeemably catholic.

\section{INDEX}

Mots-clés : écrit, catholicisme, jésuite, circulation, communauté, réseau

Keywords : writing, Catholicism, Jesuit, circulation, community, network

\section{AUTEURS}

\section{GAËLLE SERENA}

ATER

Université Toulouse 2-Le Mirail

gaelleserena@noos.fr 